\title{
Multidimensional Apathy and Executive Dysfunction in Amyotrophic Lateral Sclerosis
}

Ratko Radakovic ${ }^{12345^{*}}$, Laura Stephenson ${ }^{2}$, Judith Newton ${ }^{2}$, Christopher Crockford ${ }^{14}$, Robert Swingler ${ }^{24}$, Siddharthan Chandran ${ }^{24}$ and Sharon Abrahams ${ }^{1245}$

1 Human Cognitive Neuroscience Unit - School of Philosophy, Psychology \& Language Sciences, University of Edinburgh, Edinburgh, UK

2 Anne Rowling Regenerative Neurology Clinic, University of Edinburgh, Edinburgh, UK

3 Alzheimer Scotland Dementia Research Centre, University of Edinburgh, Edinburgh, UK

4 Euan MacDonald Centre for MND Research, University of Edinburgh, Edinburgh, UK 5 Centre for Cognitive Ageing and Cognitive Epidemiology, University of Edinburgh, Edinburgh, UK

Word Count (exc. Tables): 4972

Keywords: Apathy; Dimensional Apathy Scale; executive dysfunction; emotional processing; amyotrophic lateral sclerosis

\footnotetext{
${ }^{*}$ Correspondence to: R. Radakovic, University of Edinburgh, Department of Psychology, 7 George Square, Edinburgh, UK, EH8 9JZ

Email addresses: r.radakovic@ed.ac.uk,radakovic.ratko@gmail.com

Tel: +441316511303
} 


\begin{abstract}
Apathy and cognitive dysfunction are prominent symptoms of Amyotrophic lateral sclerosis (ALS). More specifically ALS patients show increased Initiation apathy- a lack of motivation for self-generation of thoughts as assessed by the Dimensional Apathy Scale. This study aimed to investigate the cognitive underpinnings of apathy subtypes in ALS. We hypothesized that increased Initiation apathy would be associated deficits on tests of intrinsic response generation, such as verbal fluency. We also explored the relationship of other apathy subtypes to cognitive processes, in particular emotional apathy with emotional and social cognition deficits and executive apathy with planning and goal management deficits ALS patients, and their carers $(\mathrm{N}=30)$, and healthy matched controls, and their informants $(\mathrm{N}=29)$ were recruited. All participants completed self- and informant/carer-rated Dimensional Apathy Scale, to quantify apathy subtypes (Executive, Emotional and Initiation), along with standard apathy and depression measures. Patients and controls completed the Edinburgh Cognitive and behavioural ALS Screen, and a comprehensive neuropsychological battery including emotional recognition, social cognition, intrinsic response generation tasks (verbal fluency and random number generation) and a new ecologically valid, computerised measure of planning and goal management. The results demonstrated that increased Initiation apathy was the only significantly elevated subtype in ALS (self-rated $p<.05$, informant/carer-rated $p<.01$ ). Initiation apathy was found to be significantly associated with verbal fluency deficit, while Emotional apathy was significantly associated with emotional recognition deficits. No associations were found between apathy subtypes and depression or in controls. This is the first study to show specific associations between apathy subtypes (Emotional and Initiation) and executive and emotional cognitive dysfunction, indicating possible distinct underlying mechanisms to these demotivational symptoms.
\end{abstract}




\section{Introduction}

Amyotrophic lateral sclerosis (ALS), the most common type of motor neurone disease, is a rapid, terminal neurodegenerative disease characterised by a deterioration of upper and/or lower motor neurones. Up to $50 \%$ of ALS patients also display a profile of cognitive impairments and behavioural changes (for review see Goldstein \& Abrahams, 2013). This is characterised by as executive dysfunction and social cognition deficits, with additional changes in language, while the behaviour profile is one of apathy (Abrahams, 2011, Beeldman et al., 2016).

Executive dysfunction is the most prominent cognitive impairment and presents in about $40 \%$ of ALS patients (Raaphorst, De Visser, Linssen, De Haan \& Schmand, 2010, Phukan et al., 2012; Murphy et al., 2016). This is primarily characterised by a verbal fluency deficit, with a lack of intrinsic response generation (Abrahams et al., 2000). However different types of executive function deficits have also been described where patients have difficulty in concept formation with increased errors on card sorting tests (Gordon et al., 2010, Libon et al., 2012) and impaired performance on dual tasking paradigms (Pettit et al., 2013). Furthermore, ALS patients have shown impairment on more ecologically valid executive functioning tasks of planning and goal management (Meier, Charleston \& Tippett, 2010, Štukovnik, Zidar, Podnar \& Repovš, 2010). Emotional processing and social cognition deficits have also been reported in ALS (Abrahams, 2011, Elamin, Pender, Hardiman \& Abrahams, 2012, Sedda, 2014). In one study, ALS patients rated extreme emotional stimuli more neutrally and other pleasant or unpleasant stimuli more positively (Lulé et al., 2005). When recognising emotions, ALS patients were found to perform significantly worse than controls (Zimmerman et al., 2007, Girardi et al., 2011). This deficit extends to emotional memory, where ALS patients failed to show enhanced memory for emotional material (Papps, Abrahams, Wicks, Leigh \& Goldstein, 2005, Cuddy et al., 2012). Social cognition and theory of mind deficits have also been observed, where patients have difficulty inferring what someone is feeling or thinking, (Gibbons, Snowden, Thompson, Happe, Richardson \& Neary, 2007, Cavallo, Adenzato, MacPherson, Karwig, Enrici \& Abrahams, 2011, Girardi et al., 2011, Abrahams, 2011). Two studies revealed that ALS patients were impaired in theory of mind tasks assessing understanding of social scenarios (Gibbons et al., 2007, Cavallo et al., 2011). More recent studies have used the Judgement of Preference task where 
patients have impaired mental state inference compared to controls which encompasses both affective and cognitive theory of mind (Girardi et al., 2011, van der Hulst, Bak \& Abrahams, 2015).

Behaviour change is a prominent feature of ALS, with apathy being the most frequently reported symptom occurring in around 50\% of patients (Grossman et al., 2007, Witgert et al., 2010, Girardi et al., 2011, Lillo et al., 2011) and is associated with negative prognosis (Caga et al., 2016). These studies have measured apathy as a single onedimensional construct, although it is well established that apathy is a complex syndrome composed of demotivational subtypes (Stuss, Van Reekum, \& Murphy, 2011, Marin, 1991, 1996, Levy \& Dubois, 2006, Levy 2012). The Dimensional Apathy Scale (DAS, Radakovic \& Abrahams, 2014) uses a triadic dimensional apathy framework assessing Executive, Emotional and Initiation apathy (Radakovic et al., 2016). Executive apathy, relates to a lack of motivation for attention, planning and organization, Emotional apathy, a lack of emotional motivation, as an emotional neutrality or indifference and, finally, Initiation apathy, a lack of motivation for self-generation of thoughts. Recent research has suggested that Initiation apathy is the characteristic apathy subtype observed in ALS (Radakovic et al., 2016, Santangelo et al., 2017), with a different profile of Initiation and Executive apathy being characteristic in Parkinson's disease (Radakovic, Davenport, Starr \& Abrahams, 2017). Notably the DAS also measures apathy independent of motor disability, which is important as apathy is prominent in neurodegenerative movement disorders.

Apathy and verbal fluency deficits are the most prominent features of the cognitive and behavioural profile of ALS and previous study has shown some evidence of a linkage between the two, albeit only through a unidimensional concept structure of apathy (Grossman et al., 2007). The present study aimed to delineate the cognitive processes underlying apathy in ALS. Specifically we hypothesize that it is Initiation apathy which may be linked to verbal fluency deficits as both appear to a common characteristic of the internal generation of response, thought or action (Abrahams et al., 2000, Stuss, 2011). Given that ALS patients also show difficulties with emotional and social cognition and interpreting thoughts or feelings of another individual as a theory of mind impairment, (Girardi et al., 2011, van der Hulst, Bak \& Abrahams, 2015) and also 
executive dysfunction (Meier et al., 2010, Štukovnik et al., 2010), it is possible that there may be some linkage with emotional and executive apathy, respectively.

This study aimed to investigate the cognitive underpinnings of apathy in ALS. Primarily, we hypothesised that the presence of Initiation apathy was associated with deficits on tests of intrinsic response generation such as verbal fluency, and the generation of random responses. Furthermore, secondary exploratory associations were investigated between emotional apathy and deficits in emotional and social cognition, and between executive apathy and difficulties in key executive functions of planning and goal management.

\section{Materials and Methods}

\subsection{Participants}

30 ALS patients (without dementia) and their carers were recruited from the Scottish Motor neurone disease Audit Research Trials (SMART) register. Patient's ALS Functional Rating Scale-Revised scores (Cedarbaum, Stambler, Malta, Fuller, Hilt, Thurmond, \& Nakanishi, 1999) were accessed from the SMART register. 29 healthy Controls and their informants were recruited from the University of Edinburgh Departmental Volunteer Panel. Patients and controls were excluded if they had severe disability that would hinder participation, pre-existing dementia, severe diabetes, epilepsy, alcohol/substance-related disorders, severe head injury (that required intensive care setting hospitalisation), traumatic brain injury (inclusive of subarachnoid haemorrhage) and any other significant neurological illness (such as stroke). Additionally, apathetic healthy controls were excluded from the study. Carers and informants were recruited via the patients, and healthy controls through a chainreferral sampling method.

Ethical approval was obtained from the National Health Service (NHS) South East Scotland Research Ethics Committee 01 and the School Philosophy, Psychology and Language Sciences (PPLS), University of Edinburgh Ethical Committee, in accordance with the Declaration of Helsinki. Informed consent was obtained from all participants. 
Table 1. Demographics for ALS patients $(N=30)$ and controls $(N=29)$

\begin{tabular}{|c|c|c|c|c|}
\hline & ALS Patient & Control & $\chi 2 / t / U$-value & $\begin{array}{l}\text { Patient vs } \\
\text { Control } p \text { - } \\
\text { value }\end{array}$ \\
\hline Gender (Male/Female) & $20 / 10$ & $13 / 16$ & $\chi 2=2.85$ & .09 \\
\hline Age (mean, SD) & $63.6(12.0)$ & $64.0(11.5)$ & $U=424$ & .87 \\
\hline Years of Education (mean, SD) & $13.0(2.7)$ & $14.0(3.3)$ & -1.37 & .17 \\
\hline ALSFRS-R Score (mean, SD) $)_{+}$ & $39.1(6.2)$ & & & \\
\hline Age of onset (mean, SD) years ${ }^{+\dagger}$ & $60.2(12.3)$ & & & \\
\hline $\begin{array}{l}\text { Disease duration (median, IQR) } \\
\text { months }^{+}\end{array}$ & $24(18)$ & & & \\
\hline \multicolumn{5}{|l|}{ Site of onset } \\
\hline Bulbar & $16.7 \%(N=5)$ & & & \\
\hline Limb & $83.3 \%(\mathrm{~N}=25)$ & & & \\
\hline
\end{tabular}

ALS=Amyotrophic lateral sclerosis; $S D=$ standard deviation; ALSFRS-R=ALS Functional Rating Scale-Revised; IQR=Interquartile range $\dagger \mathbf{N}=\mathbf{2 1}$

$+\dagger \mathrm{N}=\mathbf{2 5}$

There was no significant difference between patient and control groups on age, gender, and years of education (shown in table 1).

\subsection{Procedure}

ALS patients and healthy controls completed behavioural measures or questionnaires assessing their own apathy and depression. ALS carers and healthy controls' informants completed the same behavioural measures and questionnaires assessing their observation of patients' or healthy controls' apathy and depression. The questionnaires were presented in a pseudorandomised order.

The participants then completed the neuropsychological test battery composed of experimental cognitive tests, as well as background cognitive tests, with the neuropsychological battery counterbalanced so that tasks assessing similar cognitive domains did not follow on from another. These tasks were chosen because of their theoretical mapping to different apathy subtypes, where Initiation apathy related to intrinsic response generation tasks, Emotional apathy related to social cognition and emotional recognition tasks, and finally Executive apathy related to planning and goal management tasks. 
A touchscreen laptop was used for computerised neuropsychological assessment. Participants were informed that the tasks could either be completed verbally, by writing or by instructing the experimenter to complete it for them. However, if the latter option was chosen, participants were told that they should give specific instructions to the experimenter in order to complete the task.

\subsection{Assessments}

\subsubsection{Apathy and Depression}

Dimensional Apathy Scale (DAS; Radakovic \& Abrahams, 2014) was used to assess multidimensional apathy through 3 subscales assessing Executive, Emotional, and Initiation apathy. The minimum total score for each subscale is 0 (least apathetic) and the maximum 24 (most apathetic), with a total combined score of 72. The DAS was found to be valid and reliable in detecting apathy in ALS independent of physical disability (Radakovic et al., 2016).

Apathy Evaluation Scale (AES; Marin, Biedrzycki \& Firinciogullari, 1991) was used to assess unidimensional apathy. It provides one composite score, where the minimum is 18 (least apathetic) and maximum score of 72 (most apathetic). The recommended cutoffs for the self-version are based on $\geq 2$ standard deviations above the mean (Marin et al., 1991).

Geriatric Depression Scale-Short form (GDS-15; Yesavage \& Sheikh, 1986, Brown \& Schinka, 2005) was used to assess depression. The minimum score is 0 (not depressed) with the maximum being 15 (most depressed). Recommended cut-offs for the patient self-rated version are disputed but the greatest consensus in the literature is a cut-off of $>6$ for presence of depressive symptoms (Wancata, Alexandrowicz, Marquart, Weiss \& Friedrich, 2006). Both patient and informant/carer versions of the above mentioned measures were used.

\subsubsection{Background Cognitive Tests}

Test of premorbid functioning (ToPF; Wechsler, 2011) was used to assess premorbid IQ, with controls and with only those ALS patients who were able to provide verbal responses $(\mathrm{N}=24)$. 
Edinburgh Cognitive and Behavioural ALS Screen (ECAS; Abrahams et al., 2014, Niven et al., 2015) a short cognitive and behavioural assessment designed for patients with ALS and was completed by all participants, with cut-offs validated against a full neuropsychological battery of tests. The ECAS Behaviour Interview was administered to carers about their observations of the patients. ECAS scores were used to determine presence of behavioural and/or cognitive impairment based on Strong et al's (2017) proposed diagnostic criteria for ALS - frontotemporal spectrum disorder (ALS-FTSD).

\subsubsection{Experimental Cognitive Tests}

\subsubsection{Intrinsic Response Generation}

Verbal Fluency task (Abrahams et al., 2014) is incorporated as a part of the ECAS, and consists of two subtests: a free fluency test in which participants were given 1 minute (spoken) or 2 minutes (written) to produce words beginning with the letter $\mathrm{S}$ and a fixed fluency test in which participants were given 1 minute (spoken) or 2 minutes (written) to produce four letter words beginning with T. The Verbal fluency index (Vfi) was then calculated for each condition using the equations in the supplementary materials and account for motor disability. The Vfi for each condition was then converted to scores based on conversion tables provided as a part of the ECAS (Abrahams et al., 2014). The verbal fluency total score was calculated as a sum of the free fluency and fixed fluency scores conversion scores and range from 0 (impaired fluency) to 24 (unimpaired fluency).

Random Number Generation (Evans, 1978, Baddeley, Emslie, Kolodny, \& Duncan, 1998) produces an index ranged from 0 (better randomisation) and 1 (poorer randomization) for ease of analysis (Evans, 1978). Participants were asked to say or write numbers in a random fashion at a paced rate of approximately every 1 second. Due to the possibility of some ALS patients having bulbar symptomology and/or difficulty writing, which could affect their speech production and/or writing production, the pace was adjusted to between 1 and 2 seconds to accommodate. Participants were instructed to imagine that they were picking numbers out of a hat. A total of 100 numbers were recorded in a matrix and then converted into a Random Number Generation index. 


\subsubsection{Emotional Recognition and Social Cognition}

Adapted-Facial Expression of Emotion: Stimuli and Test: Ekman 60 faces test (Young, Perrett, Calder, Sprengelmeyer, \& Ekman, 2002) is a measure of emotion recognition sensitive to ALS (Zimmerman et al., 2007, Girardi et al., 2011) with the number of faces correctly recognised out of 60 and the mean intensity of emotion being recorded over experimental trials. It is a computerised task using a touch screen computer where participants were asked to judge the emotions on faces (anger, fear, disgust, sadness, surprise and happiness) over 6 practice trials and 60 experimental trials. Additionally, this task was adapted to record the intensity of emotions, on a 7-point Likert scale ranging 1 to 7 from 'very weak', 'weak', 'slightly weak', 'average', 'slightly strong', 'strong' and 'very strong', respectively.

Judgement of Preference task (Girardi et al., 2011) is a measure of theory of mind, with participants' errors recorded, based on the experimental condition and control condition. It is a computerized task using a touch screen computer where participants are presented with four picture objects (animals, colours, furniture, vegetables and fruits) in each corner of the screen. The pre-experiment condition requires participants to choose their favourite picture from different categories ( 6 trials), which is followed by the experimental condition where a drawing of a face appeared in the middle of the screen and participants were asked "Which picture does the face like the best?" (12 trials). In the control condition, a drawing of a face appeared in the middle of the screen and participants were asked to choose "Which picture is the face looking at?" (12 trials). A distractor arrow appeared for half of the trials, pointing towards different pictures to those of the face's eye gaze.

\subsubsection{Planning and Goal Management}

The Planning and Goal-management Shopping (PGS) task is a novel computerised, touch screen test that is based on two subtests from the Behavioural Assessment of Dysexecutive Syndrome - Zoo Map and Modified Six Elements subtest (Norris \& Tate, 2000).

The first part of the task (Planning shopping) measured monitoring and adjusting performance as well as planning ability. The key variables are time taken to make the 
first move and number of errors. Participants are asked to plan and complete a simulated shopping trip, which assessed independent formulation of plans as one condition and following predetermined plans as a second/control condition. It records the number of errors made, time to make the first move and time taken to complete each condition of the task. Motor disability was accounted for by adjusting the formulation of plans variable by the follow a plan variable using the equations in the supplementary materials. Detailed description procedure and calculation of the outcome scores can be found in the supplementary materials.

The second part of the task (Goal-management shopping) measured monitoring and adjusting performance, as well as organisational ability. This task recorded the overall mean deviation for time management over all subtasks and errors made that violate the limits task selection instructions. Participants are asked to manage their time of 8 minutes between 3 different types of subtasks that are likely to occur in supermarket. The Counting Money subtasks require participants to add the coins or notes shown on the screen and choose the correct answer from three options. The Sorting Shopping subtasks require participants to sort shopping items in to two bags based on the type of item. In the Customer Complaint subtasks present participants with an item bought in a shop that has been damaged and the participants are required to verbalise what the item is and what is wrong with in two to three words. They are also asked to follow certain instructions, which limit task order selection. Furthermore, the task did not record the time spent in the transition periods when changing from each subtask, as to account for motor disability in those patients in more advanced stages of ALS. Detailed description procedure, subtasks and calculation of the outcome scores can be found in the supplementary materials.

\subsection{Statistical Analysis}

$\mathrm{R}$ and SPSS statistics 21.0 were used to analyse the results. Shapiro-Wilk tests were used to assess normality and dependent on distribution of the data, t-tests, MannWhitney U tests, and Chi-square tests (all Holm corrected) were used to compare demographics, apathy scale scores, depression scores, and neuropsychological test scores of patients and controls. Kruskal-Wallis H test was used to compare patient self ratings and carer ratings on the DAS apathy subscales. Correlational analysis (Holm 
corrected Spearman's rho) was used to determine the relationship between each DAS apathy subtype and each cognitive functioning task. Additionally, partial correlational analysis (Holm corrected Spearman's rho) was performed to investigate influence of depression on observed apathy subtype- cognitive functioning task relationships. The threshold for significance was set at 0.05 .

\section{Results}

\subsection{Demographic, apathy, depression and background cognitive tests}

There was no significant difference between patients $(M=112.0, S D=6.0)$ and controls $(M=114.8, S D=8.1)$ on the ToPF, indicating that the two groups were matched on level of estimated premorbid IQ. There was a significant difference between ECAS total cognitive scores $(U=200, p=.0004)$, where patients $(\mathrm{M}=111.2, \mathrm{SD}=15.2)$ scored lower than controls $(\mathrm{M}=122.8, \mathrm{SD}=6.2) .30 \%$ of patients were below cut-off for cognitive impairment on the ECAS. The carer interview showed that the most frequently reported symptom was apathy in 55\%, followed by loss of sympathy/empathy in $48 \%$ patients.

Furthermore, based on Strong et al (2017) diagnostic criteria for ALS-FTSD, 69\% of ALS patients had cognitive and/or behavioural impairment, (28\% had behavioural impairment, $10 \%$ had cognitive impairment, $31 \%$ had ALS with cognitive and behavioural impairment). 
Table 2. Apathy and depression scores of ALS patients $(\mathrm{N}=30)$ and controls $(\mathrm{N}=29)$

\begin{tabular}{rllll}
\hline & ALS Patient & Control & \multicolumn{1}{c}{ t/U-value } & $\begin{array}{l}\text { Patient vs } \\
\text { Control } p \text { - } \\
\text { value }\end{array}$ \\
\hline DAS (self-rated) & & & & \\
Executive & $5.6(5.3)$ & $6.5(2.8)$ & $U=332$ & .24 \\
Emotional & $8.6(3.9)$ & $7.5(3.4)$ & 1.25 & .24 \\
Initiation & $12.9(5.3)$ & $9.2(2.5)$ & 3.38 & .01 \\
Executive & $5.9(5.1)$ & $3.0(2.3)$ & $U=560.5$ & .22 \\
Emotional & $10.0(3.5)$ & $7.5(4.3)$ & 2.49 & .08 \\
Initiation & $13.6(6.0)$ & $7.9(3.9)$ & $U=675.5$ & .002 \\
AES (informant/carer-rated) & $32.3(10.0)$ & $27.8(4.7)$ & $U=560$ & .22 \\
AES-Informant (mean, SD) & $33.7(10.2)$ & $26.3(5.7)$ & $U=625.5$ & $\mathbf{. 0 2}$ \\
GDS-15 self-rated (mean, SD) & $4.5(3.3)$ & $1.8(1.8)$ & $U=656$ & $\mathbf{. 0 0 5}$ \\
GDS-15 carer-rated (mean, SD) & $7.1(4.5)$ & $1.3(1.5)$ & $U=884$ & $\mathbf{. 0 0 0 0 0 1}$ \\
\hline
\end{tabular}

ALS=Amyotrophic lateral sclerosis; $S D=$ standard deviation; DAS=Dimensional Apathy Scale; AES=Apathy Evaluation Scale; GDS-15=Geriatric depression scale- Short form

Significant p-values are in bold

Table 2 shows that in relation to both self- and informant/carer-rated DAS subscales, the only significant difference between patients and controls was on Initiation apathy. There was no significant difference between ALS patients self ratings and informant/carer ratings on any of the DAS subscales. On the other scales, patients significantly differed from controls, scoring higher on the AES score (informant rating). Patients were also significantly more depressed than controls, both on the self-rated and carer-rated GDS-15.

Based on DAS abnormality cut-offs from Radakovic et al. (2016) for either self or informant/carer ratings, $60 \%(\mathrm{~N}=18)$ of patients showed at least one apathy subtype impairment. Of those half $(\mathrm{N}=9)$ of patients showed impairment on only Initiation apathy, while 3 patients showed impairment on only Emotional apathy and 3 patients showed impairment on Initiation apathy and one or two other apathy subtypes. No controls showed impairment on Initiation apathy on self or informant/carer ratings.

5 controls were excluded from the study due being impaired on the informant/carerrated DAS (3 on Emotional apathy and 2 on Executive apathy). 


\subsection{Executive functions, social cognition and emotional recognition}

Table 3. Neuropsychological assessment scores for ALS patients $(\mathrm{N}=30)$ and controls $(\mathrm{N}=\mathbf{2 9})$

\begin{tabular}{|c|c|c|c|c|}
\hline & ALS Patient & Control & t/U-value & $\begin{array}{l}\text { Patient } \\
\text { vs } \\
\text { Control } \\
p \text {-value }\end{array}$ \\
\hline \multicolumn{5}{|l|}{ Intrinsic response generation } \\
\hline Verbal fluency score total (mean, SD) & $17.7(5.9)$ & $21.7(2.0)$ & $U=215$ & .006 \\
\hline RNGi (mean, SD) & $\begin{array}{c}0.300 \\
(0.068)\end{array}$ & $\begin{array}{l}0.277 \\
(0.045)\end{array}$ & $U=582$ & .13 \\
\hline \multicolumn{5}{|l|}{$\begin{array}{l}\text { Social cognition and emotional } \\
\text { recognition }\end{array}$} \\
\hline Like best condition errors (mean, SD) & $0.50(1.28)$ & $0.00(0.00)$ & $U=582$ & .047 \\
\hline Look at condition errors (mean, SD) & $0.37(1.38)$ & $0.17(0.54)$ & $U=435.5$ & 1.00 \\
\hline \multicolumn{5}{|l|}{ Ekman 60 faces test } \\
\hline Overall correct (mean, SD) & $45.6(5.7)$ & $49.1(5.1)$ & -2.49 & .10 \\
\hline Intensity score (mean, SD) ${ }^{\ddagger}$ & $5.2(0.6)$ & $5.0(0.7)$ & 0.67 & 1.00 \\
\hline \multicolumn{5}{|l|}{ PGS Task } \\
\hline \multicolumn{5}{|l|}{ Planning shopping task } \\
\hline Planning time seconds (mean, SD) & $54.5(72.0)$ & $91.9(91.5)$ & $U=255$ & .047 \\
\hline Planning Errors (mean, SD) & $2.8(3.4)$ & $2.0(3.9)$ & $U=539$ & .41 \\
\hline \multicolumn{5}{|l|}{ Goal management shopping task } \\
\hline $\begin{array}{r}\text { Grand mean deviation seconds (mean, } \\
\text { SD) }\end{array}$ & $36.5(20.2)$ & $23.7(13.7)$ & 2.92 & .046 \\
\hline No. of consecutive errors (mean, SD) & $1.6(2.9)$ & $0.9(1.5)$ & $U=478.5$ & 1.00 \\
\hline
\end{tabular}

Vfi=Verbal fluency index; SD=standard deviation; n.s.=not significant; PGS=Planning and Goalmanagement Shopping; RNGi=Random Number Generation index ‡ Range of intensity scores is 1 (very weak) to 7 (very strong), with 4 being the 'average' intensity 扯 Lower score indicates poorer performance

¥¥ Rounded to 3 decimal places due to use of index, interpretation is closer to 0 is better randomisation and closer to 1 is poorer randomization

Significant $p$-values are in bold

Table 3 shows a profile of cognitive dysfunction in ALS patients, when compared to controls. The most notable differences between ALS patients and controls was on intrinsic response generation tasks, where patients performed significantly worse on verbal fluency. There was no significant difference in randomization on the number generation task between patients and controls.

In the Judgement of Preference task ALS patients made more errors in the like best condition, indicating a difficulty in the interpretation of thoughts of others, while there 
was no difference between patients and controls on the look at condition. In terms of emotional recognition and emotional intensity rating on the Ekman 60 faces task, ALS patients and controls did not significantly differ.

In the Planning shopping task, patients did take significantly more time to plan routes around the map in comparison to controls. Additionally, on the Goal management shopping task, ALS patients performed significantly worse at managing their time than controls between different tasks. Despite the differences in times, there were no significant differences found between patients and controls on the errors on the Planning shopping and the Goal management shopping task.

\subsection{Associations between apathy subtypes and cognitive profile}

Table 4 shows associations for both self- and informant/carer-rated apathy subtype with intrinsic response generation, emotional recognition, social cognitive tasks performance and planning and goal management tasks. Correlational analysis was only performed on those variables where ALS patients were found to be significantly different from controls based on this study's results and previous literature (Verbal fluency total score, Ekman Number Correct, Judgement of Preference Like best condition errors, Planning time and Goal Management Grand mean deviation).

Table 4. Spearman's rho correlations between DAS apathy subscales (both patient selfand informant/carer-rated) and select cognitive functioning task performances in ALS patients $(\mathrm{N}=30)$ and their carers $(\mathrm{N}=30)$

\begin{tabular}{lllllll}
\hline & & $\begin{array}{l}\text { Verb } \\
\text { Flu } \\
\text { Score }\end{array}$ & $\begin{array}{l}\text { Ekman } \\
\text { No. } \\
\text { correct }\end{array}$ & $\begin{array}{l}\text { JoP Like } \\
\text { Err }\end{array}$ & $\begin{array}{l}\text { Plan } \\
\text { time }\end{array}$ & $\begin{array}{l}\text { GM } \\
\text { Dev }\end{array}$ \\
\hline Self DAS & Initiation & $-\mathbf{0 . 5 1 *}$ & -0.27 & -0.08 & -0.37 & 0.16 \\
& Emotional & -0.33 & $-\mathbf{0 . 5 3 *}$ & 0.02 & -0.47 & 0.15 \\
& Executive & -0.33 & -0.21 & -0.02 & -0.28 & 0.09 \\
& & & & & & \\
Informant/carer & Initiation & $-\mathbf{0 . 4 5 *}$ & -0.37 & 0.01 & -0.31 & 0.13 \\
DAS & Emotional & -0.33 & -0.15 & 0.08 & -0.45 & 0.26 \\
& Executive & -0.28 & -0.21 & 0.14 & -0.24 & 0.03 \\
\hline
\end{tabular}

DAS=Dimensional Apathy Score; GM Dev= Goal Management Grand mean deviation; JoP Like Err=Judgement of Preference Task Like Best Condition Errors; Verb Flu Score=Verbal fluency total score

Significant correlations are in bold

$* p<.05$ 
In patients' self and informant/carer ratings on the DAS, the verbal fluency performance was found to be correlated with Initiation apathy, with higher Initiation apathy being associated with more impaired verbal fluency. Self-rated Emotional apathy was significantly correlation with performance on the emotional recognition on the Ekman 60 faces task, where the higher the Emotional apathy the more errors were made on the emotional recognition task. Figure 1 shows these 3 significant associations.
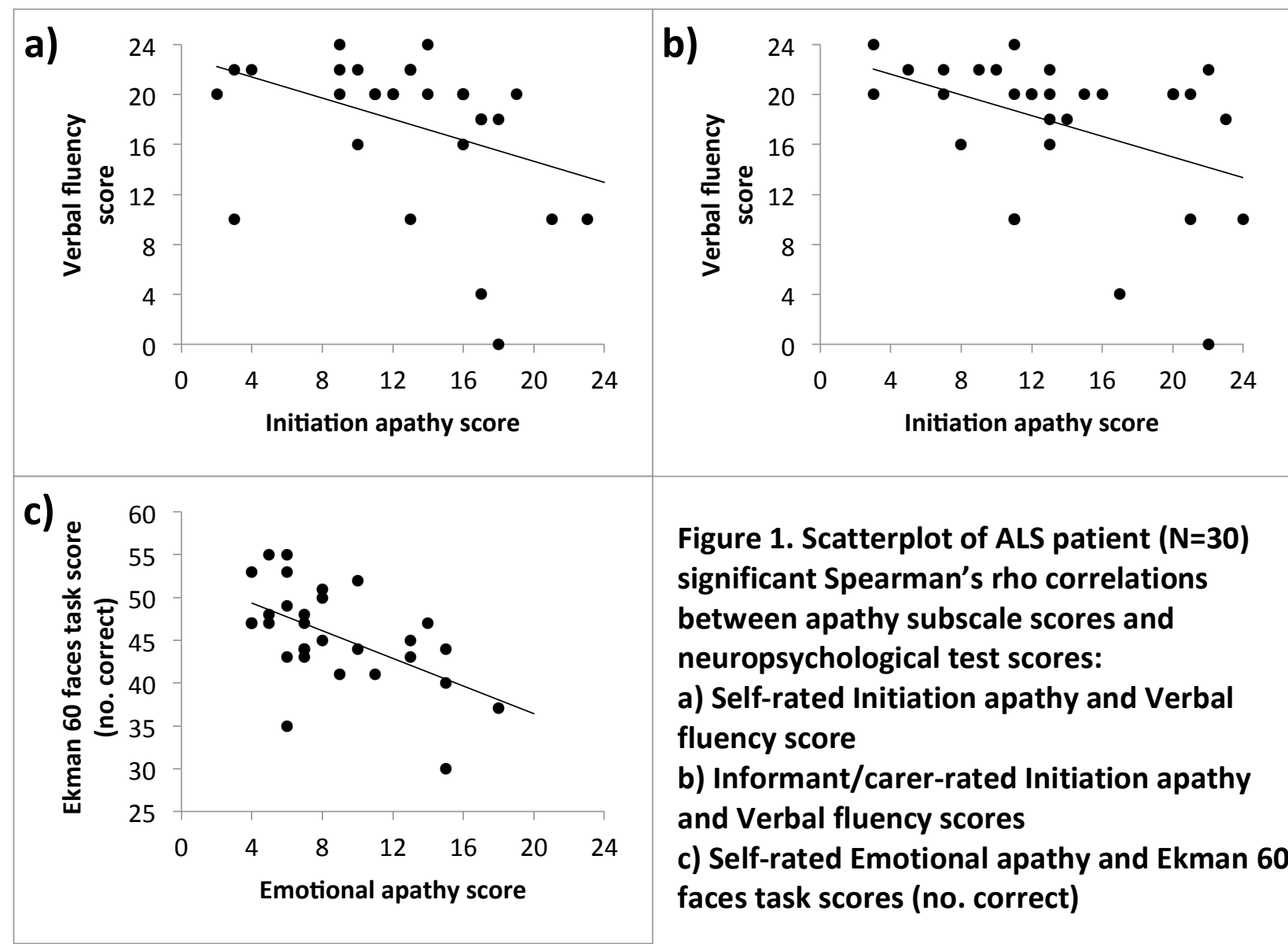

Figure 1. Scatterplot of ALS patient ( $N=30)$ significant Spearman's rho correlations between apathy subscale scores and neuropsychological test scores:

a) Self-rated Initiation apathy and Verbal fluency score b) Informant/carer-rated Initiation apathy and Verbal fluency scores c) Self-rated Emotional apathy and Ekman 60 faces task scores (no. correct)

There were no significant correlations between the Planning shopping and Goal management shopping task performance and Executive apathy. Furthermore, in ALS patients, cognitive functioning task performance associations were not found with depression. Also a partial correlational analysis controlling for depression did not change the direction or strength of the significant apathy subtype-cognitive relationships results. Additionally, these apathy subtype-cognition relationships observed in ALS were not found in controls. 


\section{Discussion}

This study has revealed some of the potential cognitive underpinnings of apathy in a neurodegenerative disease. ALS patients showed an increase in Initiation apathy, consistent with previous results (Radakovic et al., 2016, Santangelo et al., 2017). There was a significant relationship between increased Initiation apathy and poor performance on verbal fluency. There was also an association between the Emotional apathy subtype and impaired emotional recognition in ALS patients. While the verbal fluency deficit in ALS is well established (Abrahams et al., 2000), our study is the first to show apathy subtype- cognitive dysfunction associations, which due to the use of the DAS takes in to account motor disability. Previous research has only found an association between one-dimensional apathy and the verbal fluency deficit (Grossman et al., 2007) and also social cognition deficits (Girardi et al., 2011, van der Hulst, Bak \& Abrahams, 2015). This finding goes beyond this to demonstrate that it is specifically Initiation apathy which is increased in ALS and that this type of apathy may have common underlying mechanisms to the verbal fluency deficit which characterises the cognitive profile of the disorder. In relation to current cognitive models of executive functions, Stuss and colleagues propose that the key frontal lobe functions are Energization, which relates to initiating and sustaining response, Monitoring and task setting which monitor and adjust performance, Behavioral/emotional self-regulation which integrates emotional and social aspects of behaviour and Metacognition/Integration relating to higher order, coordinative processing between the other functions of the frontal lobe. Specifically, Energization deficits are often observed as decreased output during verbal fluency tasks (Stuss \& Alexander, 2007, Stuss, 2011), with recent research showing selective Energization deficits in ALS (Gillingham et al., 2017). The findings of our study suggest this may be a possible underlying process common to both Initiation apathy and verbal fluency.

The DAS apathy subtypes also have some similarities with triadic substructure of apathy suggested by Levy and Dubois (2006, Levy 2012). The component akin to Initiation apathy is termed Auto-activation apathy, and is an impairment in selfgeneration of output. The neurological basis of Auto-activation apathy has been suggested to be dysfunction of the medial prefrontal cortex extending to the cognitive 
and limbic territories of the basal ganglia (Levy \& Dubois, 2006, Levy 2012). Research in ALS has found abnormalities in similar areas, such as anterior cingulate cortex, were associated with apathy (Woolley, Zhang, Schuff, Weiner \& Katz, 2011) and significant atrophy of the caudate nucleus, which is thought to mediate motivation (Bede et al., 2013). Furthermore verbal fluency deficits have been associated with decreased bloodoxygen levels in the middle frontal gyrus and the anterior cingulate gyrus (Abrahams et al., 2004), with other structural connectivity research showing that the verbal fluency task was more dependent on the pathway integrity of the superior frontal gyrus and inferior frontal gyrus (Pettit et al., 2013), associated with neuroanatomical subtypes of apathy (Levy \& Dubois, 2006, Levy 2012).

Random number generation was also used to explore similar intrinsic response generation processes as in the verbal fluency task. This did not show that generation of both words and numbers is affected in ALS patients. However such tasks not only involve response generation, but are also dependent on inhibitory control, where individuals are unable to suppress their urge to stick to a numerical pattern when performing this task (Miyake, Friedman, Emerson, Witzki, Howerter \& Wager, 2000). Previous research showed unreliable evidence towards an impairment in random sequence generation in ALS (Abrahams et al., 1997). Verbal fluency seems to capture the intrinsic response deficit in ALS more consistently.

While patients in this study did not have impaired emotional recognition, previous research has show emotional recognition deficits (Girardi et al. 2011), specific to fear and anger recognition in ALS (Elamin, Pender, Hardiman \& Abrahams, 2012). Most notably, here it was observed that the reduced accuracy in recognising emotional expressions was significantly associated with increased Emotional apathy in ALS patients, but only in patient self ratings. The informant/carer-rated DAS Emotional apathy subscale did not significantly associate with emotional recognition. The role of the informant caring for the individual and motivating them throughout daily life, as well as the disease course, could impact their perception of apathy, as mentioned in previous research (Radakovic et al., 2017). This could make it difficult for some informant/carers to observe certain aspects of apathy. Further research would be needed to explore the impact of the caregiver role on motivation of the person with ALS 
throughout the disease. However, of note there was no difference between ALS patient self and informant/caregiver ratings over all the DAS subscales, indicative of a preserved awareness of apathy at the group level.

While Emotional apathy has not been observed to be typical in ALS, some patients do display this type of apathy (Radakovic, et al., 2016). Further, in this study $48 \%$ of the ALS patients were rated as showing a degree of loss of sympathy/empathy using the ECAS behaviour interview, in which Emotional apathy may be a factor together with impaired social cognition. While there is recent research indicating emotional empathy impairments in ALS (Cerami et al., 2014), this seems to be the first report of levels of Emotional apathy being associated with emotional recognition deficits. Similar such emotional processing deficits have been observed (Gillingham et al., 2017) which were proposed to be due to dysfunction of behavioral/emotional self-regulation (Stuss \& Alexander, 2007, Stuss, 2011). The current study suggests further shared underlying components of Emotional apathy and emotional recognition, requiring further exploration of behavioral/emotional self-regulation dysfunction. Furthermore, DAS Emotional apathy is comparable to Levy and Dubois' Emotional-affective apathy subtype where there is impairment in emotional processing. This is neurologically characterised by orbital and medial prefrontal cortex dysfunction (Levy \& Dubois, 2006, Levy 2012), which overlaps with ventromedial prefrontal cortex areas associated Stuss' behavioral/emotional self-regulation areas (Brodmann areas 13 and 14; Stuss \& Alexander, 2007, Stuss, 2011). Future research should look at the neural underpinnings of Emotional apathy in concordance with emotional recognition deficits, behavioral/emotional self-regulation and loss of sympathy/empathy.

In terms of social and emotional cognition, overall patients were impaired on all tasks to some extent when compared to controls. In the Judgement of Preference task, patients made more errors than controls in the affective ('like best') condition, showing a difficulty in inferring eye-gaze related preference, and therefore, mental state of others. This was also consistent with previous research showing ALS patients impairment in these domains of cognitive functioning (Zimmerman et al., 2007, Girardi et al., 2011, Cuddy et al., 2012). Previous research has shown that impairment in social cognition was associated with apathy as a behavioural change (Girardi et al., 2011). This study 
found no such association with any of the apathy subtypes, which could be due to the low prevalence of isolated Emotional apathy impairment in patients $(\mathrm{N}=3)$.

This study used a novel PGS task to assess planning and goal management, which was designed on the basis of well-established methodology of the Behavioural Assessment of Dysexecutive Syndrome - Zoo Map and Modified Six Elements subtest (Norris \& Tate, 2000). The task was designed to improve ecological validity by utilising an errand that many individuals have an experience with i.e. shopping. Also, the Shopping task utilised calculation of outcome specifically designed to take in to account motor disability, but still measuring effectively participants' ability to manage time and plan. This task showed that patients differed from controls in time management ability, however there were no associations between Executive apathy and either of these domains. In this study there was no overall evidence of isolated executive apathy. It may be interesting to investigate neurodegenerative diseases where Executive apathy is one of the more defining deficits such as in Parkinson's disease (Radakovic et al., 2017), which might show more impairment on these planning and goal management executive functioning tasks.

With the availability of the new proposed diagnostic criteria for ALS-FTSD (Strong et al., 2017), we found that $69 \%$ of our ALS patients showed cognitive and/or behavioural impairment. The Axis II of these diagnostic criteria has specified that the presence of apathy is a key feature of the diagnosis of ALS with behavioural impairment, similarly so the verbal fluency deficit is a key feature of diagnosis of ALS with cognitive impairment. This places emphasis on in depth assessments provided by the Dimensional Apathy Scale, to explore the complexities of behavioural and cognitive impairment both in ALS and other neurodegenerative diseases. Our findings therefore provide evidence of the importance of stratification of apathy as a syndrome, and associations with cognitive impairment. Furthermore, this creates further implications for understanding the relationship of these symptoms in ALS with cognitive and behavioural impairment, with further research needed to understand how these deficits affect everyday life of patients and their families. 
The limited sample size in this study did not allow for more predictive analysis to be performed with regards to executive dysfunction and apathy subtypes. However, the lack of association between cognitive task performance and apathy subtypes in controls, in addition to the fact that patient performance was not associated with depression, is supportive of the validity of the findings linking apathy subtypes to specific types of executive and cognitive dysfunction.

\section{Conclusions}

In conclusion, this study demonstrated that specifically increased Initiation apathy is related to verbal fluency impairment in ALS suggesting common underlying mechanisms. Emotional apathy although less typical of ALS may relate to emotional processing task deficits. Further research should attempt to directly identify the underlying cerebral mechanisms of this apathy-cognitive dysfunction overlap, through use of imaging techniques, and establish how this develops throughout disease progression.

\section{Acknowledgements}

With thanks to the MND Register, hosted by the Euan Macdonald Centre for MND Research and funded by MND Scotland. Thank you to Mr Robert Jinman for help in computerising and programming relating to The Planning and Goal-management Shopping (PGS) task. We would like to thank the patients and controls, and their families, for their participation in the study.

\section{Funding}

This work was supported by a scholarship from the Anne Rowling Regenerative Neurology Clinic, Alzheimer Scotland Dementia Research Centre and the University of Edinburgh.

\section{References}

Abrahams, S. (2011). Social cognition in amyotrophic lateral sclerosis. Neurodegenerative Disease Management, 1(5), 397-405. 
Abrahams, S., Goldstein, L. H., Simmons, A., Brammer, M., Williams, S. C. R., Giampietro, V., \& Leigh, P. N. (2004). Word retrieval in amyotrophic lateral sclerosis: a functional magnetic resonance imaging study. Brain, 127(7), 1507-1517.

Abrahams, S., Goldstein, L. H., Al-Chalabi, A., Pickering, A., Morris, R. G., Passingham, R. E., Passingham, R. E., Brooks, D. J. \& Leigh, P. N. (1997). Relation between cognitive dysfunction and pseudobulbar palsy in amyotrophic lateral sclerosis. Journal of Neurology, Neurosurgery \& Psychiatry, 62(5), 464-472.

Abrahams, S., Leigh, P. N., Harvey, A., Vythelingum, G. N., Grise, D., \& Goldstein, L. H. (2000). Verbal fluency and executive dysfunction in amyotrophic lateral sclerosis (ALS). Neuropsychologia, 38(6), 734-747.

Abrahams, S., Newton, J., Niven, E., Foley, J., \& Bak, T. H. (2014). Screening for cognition and behaviour changes in ALS. Amyotrophic Lateral Sclerosis and Frontotemporal Degeneration, 15(1-2), 9-14.

Baddeley, A. D., Emslie, H., Kolodny, J., \& Duncan, J. (1998). Random generation and the central executive of working memory. Quarterly Journal of Experimental Psychology-A, $51,819-852$.

Beeldman, E., Raaphorst, J., Twennaar, M. K., de Visser, M., Schmand, B. A., \& de Haan, R. J. (2016). The cognitive profile of ALS: a systematic review and meta-analysis update. Journal of Neurology, Neurosurgery \& Psychiatry, 87, 611-619.

Bede, P., Elamin, M., Byrne, S., McLaughlin, R. L., Kenna, K., Vajda, A., Pender, N., Bradley, D. G., \& Hardiman, O. (2013). Basal ganglia involvement in amyotrophic lateral sclerosis. Neurology, 81(24), 2107-2115.

Brown, L. M., \& Schinka, J. A. (2005). Development and initial validation of a 15-item informant version of the Geriatric Depression Scale. International Journal of Geriatric Psychiatry, 20(10), 911-918.

Caga, J., Turner, M. R., Hsieh, S., Ahmed, R. M., Devenney, E., Ramsey, E., Zoing, M. C., 
Mioshi, E. \& Kiernan, M. C. (2016). Apathy is associated with poor prognosis in amyotrophic lateral sclerosis. European journal of neurology, 23, 891-897.

Cavallo, M., Adenzato, M., MacPherson, S. E., Karwig, G., Enrici, I., \& Abrahams, S. (2011). Evidence of social understanding impairment in patients with amyotrophic lateral sclerosis. PLoS One, 6(10), e25948-e25948.

Cedarbaum, J. M., Stambler, N., Malta, E., Fuller, C., Hilt, D., Thurmond, B., \& Nakanishi, A. (1999). The ALSFRS-R: a revised ALS functional rating scale that incorporates assessments of respiratory function. Journal of the Neurological Sciences, 169(1), 13-21.

Cerami, C., Dodich, A., Canessa, N., Crespi, C., Iannaccone, S., Corbo, M., Lunetta, C., Consonni, M., Scola, E., Falini, A., \& Cappa, S. F. (2014). Emotional empathy in amyotrophic lateral sclerosis: a behavioural and voxel-based morphometry study. Amyotrophic Lateral Sclerosis and Frontotemporal Degeneration, 15(1-2), 21-29.

Cuddy, M., Papps, B. J., Thambisetty, M., Leigh, P. N., \& Goldstein, L. H. (2012). Processing and memory for emotional and neutral material in amyotrophic lateral sclerosis. Amyotrophic Lateral Sclerosis, 13(6), 592-598.

Elamin, M., Pender, N., Hardiman, O., \& Abrahams, S. (2012). Social cognition in neurodegenerative disorders: a systematic review. Journal of Neurology, Neurosurgery \& Psychiatry, 83(11), 1071-1079.

Evans, F. J. (1978). Monitoring attention deployment by random number generation: an index to measure subjective randomness. Bulletin of the Psychonomic Society, 12, 35-38.

Gibbons, Z. C., Snowden, J. S., Thompson, J. C., Happe, F., Richardson, A., \& Neary, D. (2007). Inferring thought and action in motor neurone disease. Neuropsychologia, 45(6), 1196-1207.

Gillingham, S. M., Yunusova, Y., Ganda, A., Rogaeva, E., Black, S. E., Stuss, D. T., \& Zinman, L. (2017). Assessing cognitive functioning in ALS: A focus on frontal lobe processes. Amyotrophic Lateral Sclerosis and Frontotemporal Degeneration, 18, 182-192. 
Girardi, A., MacPherson, S. E., \& Abrahams, S. (2011). Deficits in emotional and social cognition in amyotrophic lateral sclerosis. Neuropsychology, 25(1), 53-65.

Goldstein, L. H., \& Abrahams, S. (2013). Changes in cognition and behaviour in amyotrophic lateral sclerosis: nature of impairment and implications for assessment. The Lancet Neurology, 12(4), 368-380.

Gordon, P. H., Goetz, R. R., Rabkin, J. G., Dalton, K., Mcelhiney, M., Hays, A. P., Marder, K., Stern, Y., \& Mitsumoto, H. (2010). A prospective cohort study of neuropsychological test performance in ALS. Amyotrophic Lateral Sclerosis, 11(3), 312-320.

Grossman, A. B., Woolley-Levine, S., Bradley, W. G., \& Miller, R. G. (2007). Detecting neurobehavioral changes in amyotrophic lateral sclerosis. Amyotrophic Lateral Sclerosis, 8(1), 56-61.

Levy, R. (2012). Apathy: a pathology of goal-directed behaviour. A new concept of the clinic and pathophysiology of apathy. Revue Neurologique, 168(8), 585-597.

Levy, R., \& Dubois, B. (2006). Apathy and the functional anatomy of the prefrontal cortex-basal ganglia circuits. Cerebral cortex, 16(7), 916-928.

Libon, D. J., McMillan, C., Avants, B., Boller, A., Morgan, B., Burkholder, L., Chandrasekaran, K., Elman, L., McCluskey, L., \& Grossman, M. (2012). Deficits in concept formation in amyotrophic lateral sclerosis. Neuropsychology, 26(4), 422.

Lillo, P., Mioshi, E., Zoing, M. C., Kiernan, M. C., \& Hodges, J. R. (2011). How common are behavioural changes in amyotrophic lateral sclerosis?. Amyotrophic Lateral Sclerosis, 12(1), 45-51.

Lulé, D., Kurt, A., Jürgens, R., Kassubek, J., Diekmann, V., Kraft, E., Neumann, N., Ludolph, A. C., Birbaumer, N., \& Anders, S. (2005). Emotional responding in amyotrophic lateral sclerosis. Journal of Neurology, 252(12), 1517-1524. 
Marin, R. S. (1991). Apathy: a neuropsychiatric syndrome. The Journal of Neuropsychiatry and Clinical Neuroscience, 3, 243-254.

Marin, R. S. (1996). Apathy: concept, syndrome, neural mechanisms, and treatment. Seminars in Clinical Neuropsychiatry, 1, 304-314.

Marin, R. S. Biedrzycki, R. C., \& Firinciogullari, S. (1991). Reliability and validity of the Apathy Evaluation Scale. Psychiatry Research, 38(2), 143-162.

Meier, S. L., Charleston, A. J., \& Tippett, L. J. (2010). Cognitive and behavioural deficits associated with the orbitomedial prefrontal cortex in amyotrophic lateral sclerosis. Brain, 133(11), 3444-3457.

Miyake, A., Friedman, N. P., Emerson, M. J., Witzki, A. H., Howerter, A., \& Wager, T. D. (2000). The unity and diversity of executive functions and their contributions to complex "frontal lobe" tasks: A latent variable analysis. Cognitive psychology, 41(1), 49100.

Murphy, J., Factor-Litvak, P., Goetz, R., Lomen-Hoerth, C., Nagy, P. L., Hupf, J., ... \& Mitsumoto, H. (2016). Cognitive-behavioral screening reveals prevalent impairment in a large multicenter ALS cohort. Neurology, 86(9), 813-820.

Niven, E., Newton, J., Foley, J., Colville, S., Swingler, R., Chandran, S., Bak, T. H., \& Abrahams, S. (2015). Validation of the Edinburgh Cognitive and Behavioural Amyotrophic Lateral Sclerosis Screen (ECAS): A cognitive tool for motor disorders. Amyotrophic Lateral Sclerosis and Frontotemporal Degeneration, 16, 172-179.

Norris, G., \& Tate, R. L. (2000). The behavioural assessment of the dysexecutive syndrome (BADS): ecological, concurrent and construct validity. Neuropsychological Rehabilitation, 10, 33-45.

Papps, B., Abrahams, S., Wicks, P., Leigh, P. N., \& Goldstein, L. H. (2005). Changes in memory for emotional material in amyotrophic lateral sclerosis (ALS). Neuropsychologia, 43(8), 1107-1114. 
Pettit, L. D., Bastin, M. E., Smith, C., Bak, T. H., Gillingwater, T. H., \& Abrahams, S. (2013). Executive deficits, not processing speed relates to abnormalities in distinct prefrontal tracts in amyotrophic lateral sclerosis. Brain, 136, 3290-3304

Phukan, J., Elamin, M., Bede, P., Jordan, N., Gallagher, L., Byrne, S., Lynch, C., Pender, N., \& Hardiman, 0. (2012). The syndrome of cognitive impairment in amyotrophic lateral sclerosis: a population-based study. Journal of Neurology, Neurosurgery \& Psychiatry, 83(1), 102-108.

Raaphorst, J., De Visser, M., Linssen, W. H., De Haan, R. J., \& Schmand, B. (2010). The cognitive profile of amyotrophic lateral sclerosis: a meta-analysis. Amyotrophic Lateral Sclerosis, 11(1-2), 27-37.

Radakovic, R., \& Abrahams, S. (2014). Developing a new apathy measurement scale: Dimensional Apathy Scale. Psychiatry Research, 219(3), 658-663

Radakovic, R., Stephenson, L., Colville, S., Swingler, R., Chandran, S., \& Abrahams, S. (2016). Multidimensional apathy in ALS: validation of the Dimensional Apathy Scale. Journal of Neurology, Neurosurgery \& Psychiatry, 87, 663-669.

Radakovic, R., Davenport, R., Starr, J. M., \& Abrahams, S. (2017). Apathy dimensions in Parkinson's disease. International Journal of Geriatric Psychiatry. doi:10.1002/gps.4697

Santangelo, G., Siciliano, M., Trojano, L., Femiano, C., Monsurrò, M., Tedeschi, G., Trojsi, F. (2017). Apathy in Amyotrophic Lateral Sclerosis: insights from Dimensional Apathy Scale. Amyotrophic Lateral Sclerosis and Frontotemporal Degeneration. doi:10.1080/21678421.2017.1313865

Sedda, A. (2014). Disorders of emotional processing in amyotrophic lateral sclerosis. Current Opinion in Neurology, 27(6), 659-665.

Strong, M. J., Abrahams, S., Goldstein, L. H., Woolley, S., Mclaughlin, P., Snowden, J., Mioshi, E., Roberts-South, A., Benatar, M., HortobáGyi, T., Rosenfeld, J., Silani, V., Ince, P. G., \& Turner, M. R. (2017). Amyotrophic lateral sclerosis-frontotemporal spectrum 
disorder (ALS-FTSD): Revised diagnostic criteria. Amyotrophic Lateral Sclerosis and Frontotemporal Degeneration, 18(3-4), 153-174.

Stuss, D. T. (2011). Functions of the frontal lobes: relation to executive functions. Journal of the International Neuropsychological Society, 17(5), 759-765.

Stuss, D. T., \& Alexander, M. P. (2007). Is there a dysexecutive syndrome?. Philosophical Transactions of the Royal Society B: Biological Sciences, 362(1481), 901-915.

Stuss, D. T., Van Reekum, R., \& Murphy, K. J. (2011). Differentiation of states and causes of apathy. In J. C. Borod (Ed.), The Neuropsychology of Emotion (pp. 340-363), Oxford: Oxford University Press.

Štukovnik, V., Zidar, J., Podnar, S., \& Repovš, G. (2010). Amyotrophic lateral sclerosis patients show executive impairments on standard neuropsychological measures and an ecologically valid motor-free test of executive functions. Journal of clinical and experimental neuropsychology, 32(10), 1095-1109.

van der Hulst, E. J., Bak, T. H., \& Abrahams, S. (2015). Impaired affective and cognitive theory of mind and behavioural change in amyotrophic lateral sclerosis. Journal of Neurology, Neurosurgery \& Psychiatry, 86, 1208-1215.

Wancata, J., Alexandrowicz, R., Marquart, B., Weiss, M., \& Friedrich, F. (2006). The criterion validity of the Geriatric Depression Scale: a systematic review. Acta Psychiatrica Scandinavica, 114(6), 398-410.

Wechsler, D. (2011). Test of Premorbid Functioning - UK Edition. London: Pearson Assessment.

Witgert, M., Salamone, A. R., Strutt, A. M., Jawaid, A., Massman, P. J., Bradshaw, M., Mosnik. D., Appel, S. H., \& Schulz, P. E. (2010). Frontal-lobe mediated behavioral dysfunction in amyotrophic lateral sclerosis. European Journal of Neurology, 17(1), 103110. 
Woolley, S. C., Zhang, Y., Schuff, N., Weiner, M. W., \& Katz, J. S. (2011). Neuroanatomical correlates of apathy in ALS using 4 Tesla diffusion tensor MRI. Amyotrophic Lateral Sclerosis, 12(1), 52-58.

Yesavage, J. A., \& Sheikh, J. I. (1986). Geriatric Depression Scale (GDS) Recent Evidence and Development of a Shorter Version. Clinical Gerontologist, 5(1-2), 165-173.

Young, A. W., Perrett, D., Calder, A., Sprengelmeyer, R., \& Ekman, P. (2002). Facial expressions of emotions: stimuli and test (FEEST), Thames Valley Test Company, Thurstone, UK.

Zimmerman, E. K., Simmons, Z., \& Barrett, A. M. (2007). Emotional perception deficits in amyotrophic lateral sclerosis. Cognitive and behavioral neurology: official journal of the Society for Behavioral and Cognitive Neurology, 20(2), 79-82. 